\title{
On the computation of stress fields on polygonal domains with V-notches *
}

\author{
Johan Helsing $^{\dagger}$ and Anders Jonsson ${ }^{\ddagger}$
}

June 19, 2000, revised February 2, 2001

\begin{abstract}
The interior stress problem is solved numerically for a single edge notched specimen under uniaxial load. The algorithm is based on a modification of a Fredholm second kind integral equation with compact operators due to Muskhelishvili. Several singular basis functions for each of the seven corners in the geometry enable high uniform resolution of the stress field with a modest number of discretization points. As a consequence, notch stress intensity factors can be computed directly from the solution. This is an improvement over other procedures where the stress field is not resolved in the corners and where notch stress intensity factors are computed in a roundabout way via a pathindependent integral. Numerical examples illustrate the superior stability and economy of the new scheme.
\end{abstract}

Key words: Stress analysis, biharmonic equation, polygonal domain, notch stress intensity factor, corner singularity, V-notch, boundary elements, complex potentials, Muskhelishvili integral equation

\section{Introduction}

The computation of stress fields inside loaded elastic bodies with corners is a common task in mechanical and civil engineering. A corner-type of particular importance is the notch a re-entrant corner where stresses diverge and oscillate. A basic geometry involving notches and other corners is the single edge notched specimen used for fracture mechanics testing. The etching of silicon wafers is another situation where sharp notches can arise [1]. The simulation of crack propagation in perforated plates may also involve notches, since fatigue crack initiation often takes place at notch tips. Accuracy control is essential in such a simulation. The finite element method, which is the most versatile computational tool today, often give mesh-dependent results in this and other difficult settings $[2,3,4]$.

${ }^{*}$ This work was supported by NFR, TFR, and The Knut and Alice Wallenberg Foundation under TFR contracts 98-568 and 99-380.

${ }^{\dagger}$ Department of Solid Mechanics and NADA, Royal Institute of Technology, SE-100 44 Stockholm, Sweden, Email: helsing@nada.kth.se, Phone: +46-8-7907553, Fax: +46-8-4112418.

${ }^{\ddagger}$ Department of Solid Mechanics, Royal Institute of Technology, SE-100 44 Stockholm, Sweden, Email: andersj@hallf.kth.se, Phone: +46-8-7908397, Fax: +46-8-4112418. 
The reduced task of computing so-called notch stress intensity factors is also important and has relevance for strength calculations. Experiments show that simple failure criteria based on critical values of such factors exist, at least for brittle fracture $[5,6,7,8]$. This problem is extremely well conditioned. A one per cent relative change in the notch opening angle (input data) typically gives a relative change in the notch stress intensity factor (output data) of less than one per cent [9]. It could therefore be expected that notch stress intensity factors are easy to compute or that accurate benchmark results should be available. A review of the literature, which is extensive, gives the opposite impression: Authors consider the problem hard and the quality of some results can be questioned.

The main difficulty on polygonal domains, regardless of the numerical method used, is the need to resolve the stress field in the corners. The asymptotic and rather complicated behaviour of this biharmonic field has been known for almost 50 years [10]. So far no author has fully succeeded in incorporating this information into an efficient and general numerical scheme. This paper presents a way to go. Our scheme, which includes several asymptotically correct basis functions for each corner and carefully implemented quadrature, has previously been tested in work on the much simpler problem of solving Laplace's equation on polygonal domains [11].

The paper is organized as follows: Section 2 gives the classic Williams series solution for the problem of a single traction-free notch. Section 3 gives an overview of previous work. Emphasis is placed on equations and methods used. Previous numerical results for notch stress intensity factors are summarized in Table 1. In this paper, for stability reasons, we choose a Fredholm second kind integral equation with compact operators. This equation is presented in Section 4 and our particular choice of basis functions for the solution in the corners is derived in Section 5. Sections 6 and 7 concern the incorporation of these basis functions into a Nyström scheme for the solution of the integral equation. It is necessary to go into some detail here. We have tried to give enough detail so that the reader should be able to reproduce our algorithm and calculations, yet we try to be concise. Convergence plots for a notch stress intensity factor and a resolved picture of a stress field for a frequently studied setup are produced in Section 8. The objective of this section is to demonstrate the superior efficiency and stability of our scheme.

\section{The Williams expansion}

A finite, linearly elastic, specimen occupies a domain $D$. The boundary of the specimen is denoted $\Gamma$ and is given positive (counter-clockwise) orientation. Traction $\left(t_{x}^{\mathrm{pr}}, t_{y}^{\mathrm{pr}}\right)$ is prescribed on $\Gamma$. We would like to compute the stress field inside $D$.

Let $U(x, y)$ denote the Airy stress function. Since $U(x, y)$ satisfies the biharmonic equation inside $D$ it can be represented as [12]

$$
U(x, y)=\Re \mathrm{e}\{\bar{z} \phi(z)+\chi(z)\},
$$

where the potentials $\phi(z)$ and $\chi(z)$ are single-valued analytic functions of the complex variable $z=x+i y$.

Let us now consider a domain $D$ with a single traction-free notch, see Figure 1 . The origin of a cartesian coordinate system is placed at the notch tip. The notch opening angle 


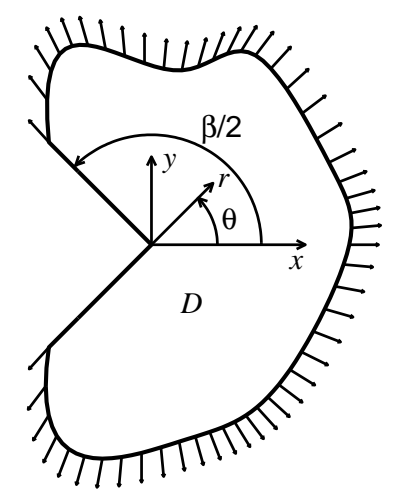

Figure 1: A domain $D$ with a traction-free notch. The notch opening angle is $\beta$. The notch tip is at the origin. Traction is applied outside the notch.

is $\beta$. With the help of variable separation in polar coordinates $r$ and $\theta$, the Airy stress function $U(r, \theta)$ in $D$ can be written as a sum of terms $U_{n}(r, \theta)$ of the form

$$
\begin{aligned}
U_{n}(r, \theta)= & \Re \mathrm{e}\left\{r^{\lambda_{n}+1}\left[a_{n} \cos \left(\left(\lambda_{n}+1\right) \theta\right)+b_{n} \cos \left(\left(\lambda_{n}-1\right) \theta\right)\right]\right. \\
& \left.+r^{\mu_{n}+1}\left[c_{n} \sin \left(\left(\mu_{n}+1\right) \theta\right)+d_{n} \sin \left(\left(\mu_{n}-1\right) \theta\right)\right]\right\} .
\end{aligned}
$$

Here $a_{n}, b_{n}, c_{n}$, and $d_{n}$ are complex coefficients determined by the applied traction, and $\lambda_{n}$ and $\mu_{n}$ are symmetric and antisymmetric exponents given by the infinite number of solutions to the trigonometric equations

$$
\begin{aligned}
& \lambda \sin (\beta)+\sin (\lambda \beta)=0, \\
& \mu \sin (\beta)-\sin (\mu \beta)=0 .
\end{aligned}
$$

The solution (2-4) is the famous Williams expansion [10], which constitutes the starting point for almost every research paper on the computation of stress fields in corners. It is worth pointing out that the solutions $\lambda$ and $\mu$ to (3) and (4) in general appear in complex conjugate pairs, which corresponds to oscillating stress fields, although some of the first $\lambda$ and $\mu$ may be real. For example, there is always one solution $\mu=1$. Furthermore, for $\beta>\pi$, the leading solutions, $\lambda_{1}$ and $\mu_{1}$, are always real and $\lambda_{1}$ is less than one, which corresponds to a diverging stress field.

Quantities of particular interest are the so-called notch stress intensity factors. The most common of these is the dimensionless mode I notch stress intensity factor $Q_{\mathrm{I}}$, defined in a cartesian coordinate system, see Figure 1, as

$$
Q_{\mathrm{I}} \equiv \lim _{x \rightarrow 0^{+}} \sqrt{2 \pi} \frac{\sigma_{y y}}{\sigma_{\text {ref }}}\left(\frac{x}{L}\right)^{1-\lambda_{1}},
$$

where $L$ is a characteristic length scale, $\sigma_{\text {ref }}$ is a reference stress, and the limit is taken along the $x$-axis. For the single edge notched specimen under uniaxial load, a common choice is to take $L$ as the width $w$ of the specimen and $\sigma_{\text {ref }}$ as the prescribed traction $t_{y}^{\mathrm{pr}}$, 


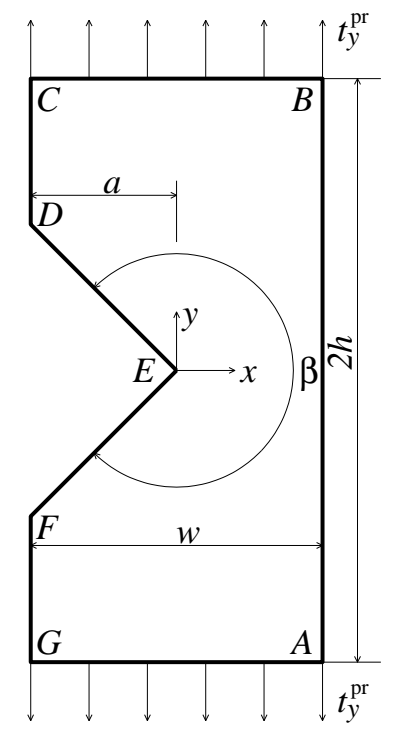

Figure 2: A generic single edge notched specimen of length $2 h$ and width $w$. The notch depth is $a$. The notch opening angle is $\beta$. Uniaxial traction $t_{y}^{\mathrm{pr}}$ is prescribed.

see Figure 2. We shall return to the computation of $Q_{\mathrm{I}}$ in more detail in Sections 3 and 6 . Experiments $[1,5,6,7,8]$ show that a simple failure criterion based on critical $Q_{\mathrm{I}}$ exists when the assumption of a linear material model is sufficient. Other failure criteria, such as point-stress and mean-stress criteria, have been proposed too. See Strandberg [8] and references therein.

For completeness we give the dimensionless mode II notch stress intensity factor $Q_{\mathrm{II}}$, defined as

$$
Q_{\mathrm{II}} \equiv \lim _{x \rightarrow 0^{+}} \sqrt{2 \pi} \frac{\sigma_{x y}}{\sigma_{\text {ref }}}\left(\frac{x}{L}\right)^{1-\mu_{1}} .
$$

\section{Review of earlier algorithms and results}

Many methods have been applied to the problem of computing stress fields and mode I notch stress intensity factors $Q_{\mathrm{I}}$ for $2 \mathrm{D}$ elastic bodies with re-entrant corners. Here follows a brief review. Reference is made to the specimen of Figure 2, when applicable. Numerical results of other authors, whenever available, are compared in Table 1 to those of the present authors, computed in Section 8.

\subsection{Spectral method calculations}

Gross and Mendelson [9] expanded the Airy stress function $U(x, y)$ for the setup in Figure 2 in a series of basis functions at the notch. Basis functions involving the 40 first solutions $\lambda$ and $\mu$ to $(3,4)$ were used. Coefficients were determined by collocation. The boundary values of $U(r, \theta)$ were satisfied in a least squares sense at 52 collocation points on $\Gamma$. Overdeterminacy was used to reduce the ill-conditioning of the formulation, which was observed for 
large opening angles $\beta$. Gross and Mendelson [9] achieved a relative error for $Q_{\mathrm{I}}$ of only $3 \cdot 10^{-4}$, see Table 1 . Few authors have reported results of better quality.

\subsection{Finite element method calculations}

A simple way to obtain $Q_{\mathrm{I}}$ with the finite element method is to use polynomial basis functions, solve the equations of elasticity, and then compute the values of $Q_{\mathrm{I}}$ by projection of the solution onto the first terms in the Williams expansion. This strategy may lead to difficulties. The stress field will be poorly resolved by the finite elements in the corners. The projection will be ill-conditioned, unless implemented with care.

Carpenter [13] used 3794 degrees of freedom on a domain involving eight corners. Up to three Williams basis functions were used in the projection. The relative error for $Q_{\mathrm{I}}$ was $4 \cdot 10^{-2}$. Dunn et al. [7] made a combined finite element method and experimental study involving single and double edge notched specimens. One Williams basis function was used in the projection. Numerical results for failure loads, based on 8032 degrees of freedom, were in $2 \%$ to $5 \%$ agreement with experiments. It is hard to say if this discrepancy is due to numerical errors, error in experimental measurements, or errors in the underlying physical model. Stability and accuracy of numerical schemes is an issue also for simple problems.

The application of a path-independent integral is an alternative to projection on basis functions. The idea goes back to Eshelby [14]. Carpenter [15, 16, 17] used the finite element method with up to 6000 degrees of freedom in combination with such an integral for the extraction of $Q_{\mathrm{I}}$. The same combination of methods was used by Atkinson et al. [18], see Table 1, and in a refined way by Sinclair et al. [19]. With 850 degrees of freedom, the latter authors report values of $Q_{\mathrm{I}}$ with a relative error of $10^{-3}$ for a setup with an analytic solution.

Yet an alternative is to seek a finite element solution away from the corners and a solution based on singular functions in regions close to the corners. The solutions can be connected with a Dirichlet-to-Neuman map. Givoli and Rivkin [20] tried this approach for a variety of fracture mechanics problems. For the setup in Figure 2 they reported only "partial success" and no results were presented. Another option is the use of tailormade finite elements. Lin and Tong [2] chose an assumed-stress hybrid formulation for the setup in Figure 2. Up to sixteen Williams basis functions were used, at the notch only, in combination with bilinear and quadratic basis functions. The results were reported to be mesh-dependent. Nevertheless, estimates for $Q_{\mathrm{I}}$ with a relative error of $2 \cdot 10^{-3}$ were obtained using 58 degrees of freedom, see Table 1. A similar method used by Yosibash and Schiff [21] gave slightly poorer results, see Table 1.

\subsection{Integral equation method calculations}

Methods for solving elastostatic problems based on integral equations can be characterized by two criteria: the choice of the integral equation and the choice of the solution technique.

Barone and Robinson [22] chose a singular integral equation based on displacements, quadratic basis functions, and up to 11 Williams basis functions for the stress-free corners of a circular plate with a $\beta=3 \pi / 2$ notch. The authors used 57 degrees of freedom and presented pointwise values for stress with four digits. Theocaris and Ioakimidis [24] chose a singular integral equation in a Nyström scheme and presented three digit results for $Q_{\mathrm{I}}$ 
Table 1: Numerical results for $Q_{\mathrm{I}}$ reported by different authors. The notch opening angle is $\beta=3 \pi / 2$. The relative notch depth is $a / w=0.4$ or $a / w=0.5$. The height to width ratio is $h / w=1, h / w=1.4$ or $h / w=3$. The numbers have been normalized for comparison.

\begin{tabular}{|l|l|r|r|}
\multicolumn{1}{c}{ Authors } & \multicolumn{1}{c}{$h / w$} & \multicolumn{1}{c}{$a / w$} \\
\hline 2.882 & Lin and Tong [2] (1980) & 1 & 0.4 \\
2.8106 & Atkinson et al. $[18](1988)$ & 1 & 0.4 \\
2.858 & Yosibash and Schiff [21] (1993) & 1 & 0.4 \\
2.858 & Lim et al. [23] (2002) & 1 & 0.4 \\
2.887773534376 & The present authors & 1 & 0.4 \\
2.888 & Gross and Mendelson [9] (1972) & 1.4 & 0.4 \\
2.886784004297 & The present authors & 1.4 & 0.4 \\
4.289 & Lin and Tong [2] (1980) & 1 & 0.5 \\
4.294 & Portela et al. $[25](1991)$ & 1 & 0.5 \\
4.2959 & Strandberg [27] (1999) & 1 & 0.5 \\
4.295886967699 & The present authors & 1 & 0.5 \\
4.297 & Gross and Mendelson [9] (1972) & 1.4 & 0.5 \\
4.295930812312 & The present authors & 1.4 & 0.5 \\
4.294 & Chen [26] (1995) & 3 & 0.5 \\
4.29592686855 & The present authors & 3 & 0.5 \\
\hline
\end{tabular}

at a notch in a semi-infinite strip. A single weight function was factored out to capture the leading behaviour of the solution at the notch tip. Portela et al. [25] chose an approach similar to that of Barone and Robinson [22] for a single edge notched specimen. Only the first symmetric and antisymmetric Williams functions at the notch were incorporated. A mesh of 16 boundary elements gave a relative error in $Q_{\mathrm{I}}$ of $4 \cdot 10^{-4}$. Chen [26], with a similar method and a total of 324 unknowns at each notch flank, also got a relative error in $Q_{\mathrm{I}}$ of $4 \cdot 10^{-4}$. See Table 1 .

Strandberg [27] chose a classic integral equation of Fredholm's second kind called the Sherman-Lauricella equation, see paragraph 56 of Mikhlin [28] and paragraph 19 of Parton and Perlin [29]. Values of $Q_{\mathrm{I}}$ for single edge notched specimens were computed with relative errors of the order of $10^{-6}$, see Table 1 . The solution technique included a Nyström scheme, a posteriori adaptive Gaussian quadrature, 1840 discretization points, and the GMRES iterative solver [30]. The notch stress intensity factor $Q_{\mathrm{I}}$ was computed via a path-independent integral.

\subsection{Other methods}

The difficulties traditionally associated with the numerical computation of notch stress intensity factors $Q_{\mathrm{I}}$ have generated a development of approximate handbook-type formulas based on interpolation between known results. See Hart [31], Ioakimidis and Theocaris [32], Bangji et al. [33], and Strandberg [34] for examples. Photoelastic methods can be used to obtain notch stress intensity factors, as demonstrated by Murthy and Rao [35]. These 
authors measured values for $Q_{\mathrm{I}}$ with a relative error of $7 \cdot 10^{-2}$ for a single edge notched specimen with relative notch depth $a / w=0.15$ and a height to width ratio of $h / w=5$.

\section{A modified Muskhelishvili equation}

Let us go back to the representation (1) for the Airy stress function and use it to find an integral equation for the interior stress problem. The classic treatment leads to the Sherman-Lauricella equation, see paragraph 56 of Mikhlin [28] and paragraph 19 of Parton and Perlin [29]. Here we choose a recent modification [36] of the Muskhelishvili equation [12] which we consider more efficient, see Remark 4.1 below. Starting with the representation for $\Psi(z)=\chi^{\prime \prime}(z)$ in terms of $\Phi(z)=\phi^{\prime}(z)$

$$
\Psi(z)=-\frac{1}{2 \pi i} \int_{\Gamma} \frac{\overline{\Phi(\tau)} \mathrm{d} \bar{\tau}}{(\tau-z)}-\frac{1}{2 \pi i} \int_{\Gamma} \frac{\bar{\tau} \Phi(\tau) \mathrm{d} \tau}{(\tau-z)^{2}}-\frac{1}{2 \pi i} \int_{\Gamma} \frac{\bar{n} \bar{t} \mathrm{~d} \tau}{(\tau-z)}, \quad z \in D,
$$

the following Fredholm second kind integral equation for the interior stress problem can be derived, see Remark 4.2,

$$
\left(I-M_{3}+i Q_{2}\right) \Phi(z)=\frac{\bar{n} t}{2}+\frac{\bar{n}}{n} \frac{1}{2 \pi i} \int_{\Gamma} \frac{n t \mathrm{~d} \bar{\tau}}{(\bar{\tau}-\bar{z})}, \quad z \in \Gamma .
$$

In (7) $M_{3}$ is a compact integral operator, see Remark 4.3 , given by

$$
M_{3} \Phi(z)=\frac{1}{2 \pi i}\left[\int_{\Gamma} \frac{\Phi(\tau) \mathrm{d} \tau}{(\tau-z)}+\frac{\bar{n}}{n} \int_{\Gamma} \frac{\Phi(\tau) \mathrm{d} \tau}{(\bar{\tau}-\bar{z})}+\int_{\Gamma} \frac{\overline{\Phi(\tau)} \mathrm{d} \bar{\tau}}{(\bar{\tau}-\bar{z})}+\frac{\bar{n}}{n} \int_{\Gamma} \frac{(\tau-z) \overline{\Phi(\tau)} \mathrm{d} \bar{\tau}}{(\bar{\tau}-\bar{z})^{2}}\right], \quad z \in \Gamma,
$$

where $n=n_{x}+i n_{y}$ is the outward unit normal vector on $\Gamma$. The operator $Q_{2}$ is a mapping from $\Gamma$ to $\mathbb{R}$, defined by

$$
Q_{2} \Phi=-\frac{1}{2 V} \Re \mathrm{e}\left\{\int_{\Gamma} \Phi(\tau) \bar{\tau} \mathrm{d} \tau\right\}
$$

where $V$ is the area of the specimen. The right hand side of (7) contains the load $t=t_{x}^{\mathrm{pr}}+i t_{y}^{\mathrm{pr}}$ along $\Gamma$. For brevity we have omitted the arguments of $n$ and $t$ in (6-8). Whenever $n$ and $t$ appear under an integral, they are functions of $\tau$. Otherwise they are functions of $z$.

In this paper it is assumed that the solvability conditions

$$
\int_{\Gamma} t \mathrm{~d} s=0
$$

where $\mathrm{d} s$ is an infinitesimal arc length $(\mathrm{d} z=i n \mathrm{~d} s)$ and

$$
Q_{2} \bar{n} t=0,
$$

which state that the resultant vector and moment of external forces are equal to zero, hold. See Remark 4.4 for details on the existence and uniqueness of the solution to (7).

Once (7) is solved, the components of the stress tensor inside the specimen can be computed [12] via

$$
\Phi(z)=\frac{1}{2 \pi i} \int_{\Gamma} \frac{\Phi(\tau) \mathrm{d} \tau}{(\tau-z)}, \quad z \in D
$$


equation (6), and

$$
\begin{gathered}
\sigma_{x x}+\sigma_{y y}=4 \Re \mathrm{e}\{\Phi(z)\}, \\
\sigma_{y y}-\sigma_{x x}+2 i \sigma_{x y}=2\left(\bar{z} \Phi^{\prime}(z)+\Psi(z)\right) .
\end{gathered}
$$

Remark 4.1 The Sherman-Lauricella equation has, in the past, been a popular choice for stress problems $[27,37,38]$. This equation is considered simpler than the Muskhelishvili equation for multply connected domains and for exterior problems, see p. 398 and 401 of Muskhelishvili [12], p. 249 and p. 255 of Mikhlin [28], and p. 158 of Parton and Perlin [29]. We will now explain why we prefer our modified Muskhelishvili equation over the Sherman-Lauricella equation (for interior problems). First we note that both the Muskhelishvili equation and the Sherman-Lauricella equations are based on the potentials $\phi(z)$ and $\psi(z)$, that they require extra operators containing arbitrarily placed points in order to have unique solutions, and that both equations are of Fredholm's second kind with compact operators. The major difference between the equations is that the unknown quantity in the Muskhelishvili equation is the limit of the analytic function $\phi(z)$ on $\Gamma$, while the unknown in the Sherman-Lauricella equation is a quantity related to $\phi(z)$ on $\Gamma$ via a Cauchy integral. The Muskhelishvili equation thus allows for the use of a numerical scheme which takes the (known) asymptotic properties of the solution into account in a simple fashion. The Muskhelishvili equation, in addition, leads to more stable post-processing, should one want to compute quantities related to $\phi(z)$ on $\Gamma$. Reasons that we use a modified Muskhelishvili equation based on the potential $\Phi(z)$, rather than the original equation based on the potential $\phi(z)$, are that quantities related to stress can be computed more accurately from $\Phi(z)$ than from $\phi(z)$, and that we can replace the operator containing an arbitrarily placed point with the operator $Q_{2}$. This gives a more stable numerical scheme [36]. Finally it could be mentioned that Fredholm equations based on the representation (6) for $\Psi(z)$, used in the modified Muskhelishvili equation, have successfully been applied to stress problems on infinite domains involving cracks and inclusions [39], interface cracks [40], and contact problems [41]. A unified approach in terms of potential representations may lead to simplifications as codes for large-scale computations, involving complicated topologies, are constructed.

Remark 4.2 The potential $\Phi(z)$ is analytic inside $D$, but its limiting value on $\Gamma$, which is the unknown quantity in (7), may not be differentiable (along $\Gamma$ in the corners) and may not even be finite (in the corners), see Sections 2 and 5. The right hand side of (7) may have similar properties, depending on the applied load. Even though (7) is originally derived under the assumption of a smooth $\Gamma$ and for the more regular potential $\phi(z)$, the equation, interpreted in a suitably generalized sense, also holds for domains with corners, see paragraphs 99 and 100 of Muskhelishvili [12]. More precisely, the corner points should be excluded from the domain of validity and integrals for which bounded primitive functions exist should be interpreted as generalized Riemann integrals.

Remark 4.3 Each of the four integrals appearing in (8) are singular and should be interpreted in Cauchy principal value sense. Note, however, that no Cauchy principal values need to be computed in the evaluation of $M_{3} \Phi(z)$. This is so since the sum of the kernels in the first and in the second integral of $M_{3}$ have the same (finite) left- and right limits 
as $\tau \rightarrow z$, at least for twice differentiable curves. Similarly, the sum of the kernels in the third and in the fourth integral of $M_{3}$ have the same (finite) left- and right limits as $\tau \rightarrow z$. Therefore the kernel of the operator $M_{3}$ can be regarded as continuous.

Remark 4.4 The potential $\Phi(z)$ for the interior stress problem is only determined to within a purely imaginary constant, and $\phi(z)$ is consequently determined to within a term containing one complex and one imaginary constant, see p. 179 of Mikhlin [28]. In (7) this indeterminacy is removed, and the solution is made unique, by our operator $Q_{2}$ [36]. It can be noted that while the interior stress problem in general is not solvable unless the conditions (10) and (11) hold, our equation (7) is solvable whatever the load $t$ may be. Uniqueness implies existence for Fredholm second kind equations.

\section{The nature of $\Phi(z)$ in a traction-free corner}

This section shows how to convert the Williams expansion of Section 2 into basis functions for the unknown potential $\Phi(z)$ of $(7)$ in a corner. Using the notation $z=r e^{i \theta}$ we can rewrite (2) as

$$
\begin{aligned}
& U_{n}(x, y)=\Re \mathrm{e}\left\{\bar{z}\left(b_{n} z^{\lambda_{n}}+\bar{b}_{n} z^{\bar{\lambda}_{n}}\right)-i \bar{z}\left(d_{n} z^{\mu_{n}}+\bar{d}_{n} z^{\bar{\mu}_{n}}\right)\right. \\
& \left.+\left(a_{n} z^{\lambda_{n}+1}+\bar{a}_{n} z^{\bar{\lambda}_{n}+1}\right)-i\left(c_{n} z^{\mu_{n}+1}+\bar{c}_{n} z^{\bar{\mu}_{n}+1}\right)\right\} / 2 .
\end{aligned}
$$

Identification of (15) with (1) and the relation $\Phi(z)=\phi^{\prime}(z)$ leads us to the conclusion that $\Phi(z)$ in a traction-free corner can be represented by a linear combination of symmetric basis functions of the form

$$
\Phi_{n}(z)_{\mathrm{symm}}=f_{n} z^{\lambda_{n}-1}+\bar{f}_{n} z^{\bar{\lambda}_{n}-1},
$$

and antisymmetric basis functions of the form

$$
\Phi_{n}(z)_{\text {antisymm }}=g_{n} z^{\mu_{n}-1}-\bar{g}_{n} z^{\bar{\mu}_{n}-1},
$$

where $f_{n}$ and $g_{n}$ are new complex coefficients. The special case of real $\lambda_{n}$ and $\mu_{n}$ renders $f_{n}$ real and $g_{n}$ purely imaginary. The symmetry properties of $\Phi_{n}(z)_{\text {symm }}$ and $\Phi_{n}(z)_{\text {antisymm }}$ with respect to the corner legs in Figure 3, right image, are the following: $\Phi_{n}(z)_{\text {symm }}$ has a symmetric real part and an antisymmetric imaginary part. $\Phi_{n}(z)_{\text {antisymm has an }}$ antisymmetric real part and a symmetric imaginary part.

\section{Two representations for $\Phi(\tau)$ on corner panels}

In Section 8 we shall solve (7) for the unknown $\Phi(\tau)$ and for the setup in Figure 2. The variable $\tau$ is preferred as argument for $\Phi$ when we refer to its limit of on $\Gamma$ in the context of integration. We shall use a Nyström scheme and eight-point composite quadrature. Quadrature panels which contain corners are called corner panels. Quadrature panels which do not contain corners are called regular panels. See Figure 3 for an illustration of how we place the eight quadrature points on corner panels and on regular panels. A Nyström scheme means that $\Phi(\tau)$ is represented by pointwise values on $\Gamma$ and that (7) should be 


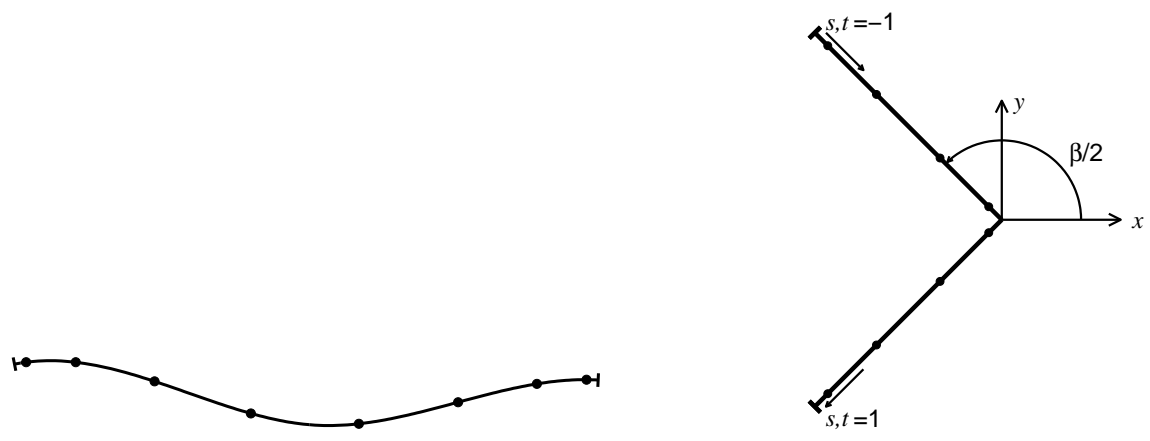

Figure 3: Left, a regular quadrature panel. Right, a corner quadrature panel with two legs. The dots symbolize points where the solution $\Phi(\tau)$ has support.

satisfied at those same points. We adopt the term source points for the points $\tau$ where $\Phi(\tau)$ has support, and the term target points for the points $z$ where (7) should be satisfied. Obviously, source points and target points are the same in a Nyström scheme. Still, it helps to distinguish between the two uses of the points when we discuss quadrature techniques below.

A delicate question is what quadrature rule to use for the action of the operator $M_{3}$ of $(8)$ on $\Phi(\tau)$ for source points lying on corner panels. Clearly, standard Gauss-Legendre quadrature will not be a good choice on corner panels since $\Phi(\tau)$ is poorly approximated by a polynomial there. We saw in Section 5 that $\Phi(\tau)$ may contain both diverging and oscillatory components. Neither is it possible to represent $\Phi(\tau)$ on corner panels as a product of a single weight function and a smooth remainder, an approach which works well for internal crack problems.

The basic idea of this paper is to introduce a second, simultaneous, representation for $\Phi(\tau)$ on corner panels. In addition to the pointwise representation for $\Phi(\tau)$ required by the Nyström scheme we will use a representation in terms of local basis functions of the type (16) and (17). The relation between the two representations is given by

$$
\begin{gathered}
\Phi\left(\tau_{j}\right)=\sum_{n=1}^{4+m_{1}} \Re \mathrm{e}\left\{f_{n}\right\}\left(\tau_{* j}^{\lambda_{n}-1}+\tau_{* j}^{\bar{\lambda}_{n}-1}\right)+\sum_{n=1+m_{2}}^{4+m_{2}-m_{1}} i \Im \mathrm{m}\left\{f_{n}\right\}\left(\tau_{* j}^{\lambda_{n}-1}-\tau_{* j}^{\bar{\lambda}_{n}-1}\right) \\
+\sum_{n=1+m_{4}}^{4+m_{4}-m_{3}} \Re \mathrm{e}\left\{g_{n}\right\}\left(\tau_{* j}^{\mu_{n}-1}-\tau_{* j}^{\bar{\mu}_{n}-1}\right)+\sum_{n=1}^{4+m_{3}} i \Im \mathrm{m}\left\{g_{n}\right\}\left(\tau_{* j}^{\mu_{n}-1}+\tau_{* j}^{\bar{\mu}_{n}-1}\right), \quad j=1,2, \ldots, 8,
\end{gathered}
$$

where $\tau_{j}$ are global coordinates, $\tau_{* j}$ are local coordinates for the eight quadrature points on a corner panel, see Figure 3 , and $m_{2}$ and $m_{4}$ denote the number of exponents $\lambda_{n}$ and $\mu_{n}$ which are real. The numbers $m_{1}$ and $m_{3}$ are chosen so that $4+m_{1} \approx 4+m_{2}-m_{1}$ and $4+m_{3} \approx 4+m_{4}-m_{3}$, that is, so that the series for real and imaginary parts of $f$ and $g$ are truncated after approximately the same number of terms. Equation (18) defines a mapping from eight complex pointwise values of $\Phi(\tau)$, on a corner panel, to sixteen real numbers $\Re \mathrm{e}\left\{f_{n}\right\}, \Im \mathrm{m}\left\{f_{n}\right\}, \Re \mathrm{e}\left\{g_{n}\right\}$, and $\Im \mathrm{m}\left\{g_{n}\right\}$. We denote this mapping $A$. Once the mapping $A$ is known it is possible to perform product integration for the convolution of the kernel $M_{3}$ 
with the function $\Phi(\tau)$, expressed of the form of (18), when source points of $\Phi(\tau)$ are on a corner panel.

There are two problems associated with the mapping $A$ of (18). First, the mapping $A$ can be very ill-conditioned for corners with a small opening angle $\beta$. The exponents $\lambda_{n}$ and $\mu_{n}$ form a rapidly growing sequence. Second, it is assumed that the corner is stress-free for the representation (18) to be valid. This assumption does not hold on corners where external stress is applied. To overcome both these problems we add to the sequence of exponents $\lambda_{n}$ and $\mu_{n}$ given by (3) and (4) a sequence of positive integers. We adopt the rule of including, in the series in (18), values of $\lambda_{n}$ and $\mu_{n}$ up to a magnitude of ten. Then we complete this series with the integers $1,2,3, \ldots$ until we get a system involving sixteen real numbers $\Re \mathrm{e}\left\{f_{n}\right\}, \Im \mathrm{m}\left\{f_{n}\right\}, \Re \mathrm{e}\left\{g_{n}\right\}$, and $\Im \mathrm{m}\left\{g_{n}\right\}$. In this way the condition number of $A$ was at most of the order of $10^{3}$.

With the help of the quantities defined in this section we can give a more operational definition of the mode I notch stress intensity factor $Q_{\mathrm{I}}$. For the single edge notched specimen of Figure 2 we have

$$
Q_{\mathrm{I}}=\frac{f_{1}}{t_{y}^{\mathrm{pr}}} 2 \sqrt{2 \pi}\left(\lambda_{1}+1\right)\left(\frac{l_{\mathrm{cp}}}{w}\right)^{1-\lambda_{1}}\left[1-\frac{\cos \left(\left(\lambda_{1}-1\right) \beta / 2\right)}{\cos \left(\left(\lambda_{1}+1\right) \beta / 2\right)}\right],
$$

where $l_{\mathrm{cp}}$ is the length of one leg of the corner panel in Figure 3, right image. Under unsymmetric conditions we obtain a mode II notch stress intensity factor $Q_{\mathrm{II}} \neq 0$ as

$$
Q_{\mathrm{II}}=\frac{\Im \mathrm{m}\left\{g_{1}\right\}}{t_{y}^{\mathrm{pr}}} 2 \sqrt{2 \pi}\left(\frac{l_{\mathrm{cp}}}{w}\right)^{1-\mu_{1}}\left[\mu_{1}-1-\left(\mu_{1}+1\right) \frac{\sin \left(\left(\mu_{1}-1\right) \beta / 2\right)}{\sin \left(\left(\mu_{1}+1\right) \beta / 2\right)}\right] .
$$

\section{$7 \quad$ The evaluation of $M_{3} \Phi(z)$}

This section discusses, in detail, how the action of the operator $M_{3}$ on $\Phi(\tau)$ is calculated. We have already noted that the potential $\Phi(\tau)$ is not smooth in the corners. Neither is the kernel of $M_{3}$ smooth. This calls for special care in the choice of quadrature. We believe that the single most important factor controlling the stability of an integral equation based numerical scheme for stress problems inside polygonal domains is the care with which the quadratures are performed. This is even more important than the particular choice of the integral equation. Below we show how our quadratures are chosen for various locations of source points of $\Phi(\tau)$ and target points of $M_{3}$.

\subsection{Regular interaction}

Straight-forward evaluation takes place when the source points of $\Phi(\tau)$ are situated on regular quadrature panels. Here $\Phi(\tau)$ is smooth. Eight-point Gauss-Legendre quadrature gives 15th order accurate evaluation of the convolution $M_{3} \Phi(z)$. For most situations this quadrature can give any desired accuracy, provided the mesh is sufficiently refined. There is, however, one important exception which arises on meshes where all quadrature panels have approximately the same length. Assume that, on such a uniform mesh, the source points of $\Phi(\tau)$ are situated on a regular panel neighbouring to, say, the first leg of a corner panel, and that the target points of $M_{3}$ are situated on the second leg of that same corner 
panel. Now 15th order quadrature may never be enough, if high accuracy is required, no matter how much the mesh is refined. This is so because of two reasons. First, $\Phi(\tau)$ on the regular panel closest to a corner panel may never be well resolved. Upon uniform refinement of the mesh, the neighbouring panel moves closer to the corner tip and $\Phi(\tau)$ assumes a more singular-like behaviour. Second, the kernel of $M_{3}$, which is known analytically, may not be well resolved. Neither here will increased uniform resolution of the entire mesh help. The kernel of $M_{3}$ is invariant under scaling in a corner (assuming a wedge-shaped corner). This situation, which can never happen on a smooth boundary, can be taken care of by letting the regular panels closest to a corner panel be smaller than the corner panel.

\subsection{Implementation of the mapping $A$}

The most difficult situations occur when the source points of $\Phi(\tau)$ are situated on a corner panel. Before we go into detail we review the implementation of the mapping $A$, which maps eight complex pointwise values of $\Phi(\tau)$ on a corner panel to sixteen real coefficients $\Re$ e $\left\{f_{n}\right\}$, $\Im \mathrm{m}\left\{f_{n}\right\}, \Re \mathrm{e}\left\{g_{n}\right\}$, and $\Im \mathrm{m}\left\{g_{n}\right\}$. The mapping $A$ is in part computed prior to running the main program. First a corner panel with two legs of unit length is considered. A mapping from eight symmetric coefficients $\Re \mathrm{e}\left\{f_{n}\right\}$ and $\Im \mathrm{m}\left\{f_{n}\right\}$ to four complex pointwise values on the first leg of the corner panel is computed as a real valued eight by eight matrix. This matrix is inverted. A similar matrix mapping from eight antisymmetric coefficients $\Re \mathrm{e}\left\{g_{n}\right\}$ and $\Im \mathrm{m}\left\{g_{n}\right\}$ to four complex pointwise values on the first leg is also computed and inverted. Then a mapping from eight complex pointwise values on the entire corner panel to four symmetric complex pointwise values and to four antisymmetric complex pointwise values on the first leg is implemented. A composition of these matrices gives the mapping $A$. See Section 5 for the meaning of symmetric and antisymmetric in this context.

\subsection{Self-interaction on corner panels}

We now consider the case of source points of $\Phi(\tau)$ and target points of $M_{3}$ both located on the same corner panel. The potential $\Phi(\tau)$ is represented in terms of local basis functions $\tau_{*}^{\lambda_{n}-1}, \tau_{*}^{\bar{\lambda}_{n}-1}, \tau_{*}^{\mu_{n}-1}$, and $\tau_{*}^{\bar{\mu}_{n}-1}$, and coefficients $f_{n}$, and $g_{n}$, computed from pointwise values via the mapping $A$.

First we observe that each corner panel has two legs of equal length, see Figure 3. We introduce a parameterization $t$ for the local coordinate $\tau_{*}$ and $s$ for the local coordinate $z_{*}$ in the coordinate system of Figure 3. Now $\tau_{*}$ and $z_{*}$ range from $e^{i \beta / 2}$ to $e^{-i \beta / 2}$ as $t$ and $s$ range from -1 to 1 . Note that $M_{3} \Phi(z)$ is invariant under scalings, translations, and rotations of the coordinate system. For this reason we can replace the global coordinates $\tau$ and $z$ with local coordinates $\tau_{*}$ and $z_{*}$ during the computation of $M_{3} \Phi(z)$ on a corner panel. A simple calculation shows that for local basis functions and target points on the same leg, the contribution to $M_{3} \Phi(z)$ is zero.

We now look at contributions to $M_{3} \Phi(z)$ from local basis functions to target points on opposite legs. Let us study the function $\tau_{*}^{\lambda_{n}-1}$. Contributions from other basis functions are computed in a similar manner. For basis functions on the second leg and target points on the first leg we have the relations $z_{*}=i s n_{z}$ and $\tau_{*}=i t n_{\tau}$, where $n_{z}=i e^{i \beta / 2}$ and 
$n_{\tau}=-i e^{-i \beta / 2}$. Insertion into (8) gives

$$
\begin{gathered}
M_{3} \tau_{*}^{\lambda_{n}-1}(z)=e^{-i \beta\left(\lambda_{n}-1\right) / 2}\left[\frac{\left(1-\bar{n}_{z}^{2} n_{\tau}^{2}\right)}{2 \pi i} \int_{0}^{1} \frac{t^{\lambda_{n}} \mathrm{~d} t}{\left(t^{2}+s^{2}-2 \Re \mathrm{e}\left\{\bar{n}_{z} n_{\tau}\right\} t s\right)}\right] \\
+e^{i \beta\left(\bar{\lambda}_{n}-1\right) / 2}\left[\frac{\left(1-\bar{n}_{z}^{2} n_{\tau}^{2}\right)}{2 \pi i} \int_{0}^{1} \frac{t^{\bar{\lambda}_{n}} \mathrm{~d} t}{\left(t^{2}+s^{2}-2 \bar{n}_{z} n_{\tau} t s\right)}\right], \quad-1 \leq s \leq 0 .
\end{gathered}
$$

Similarly, for basis functions on the first leg and target points on the second leg we get

$$
\begin{gathered}
M_{3} \tau_{*}^{\lambda_{n}-1}(z)=-e^{i \beta\left(\lambda_{n}-1\right) / 2}\left[\frac{\left(1-\bar{n}_{z}^{2} n_{\tau}^{2}\right)}{2 \pi i} \int_{0}^{1} \frac{t^{\lambda_{n}} \mathrm{~d} t}{\left(t^{2}+s^{2}+2 \Re \mathrm{e}\left\{\bar{n}_{z} n_{\tau}\right\} t s\right)}\right] \\
-e^{-i \beta\left(\bar{\lambda}_{n}-1\right) / 2}\left[\frac{\left(1-\bar{n}_{z}^{2} n_{\tau}^{2}\right)}{2 \pi i} \int_{0}^{1} \frac{t^{\bar{\lambda}_{n}} \mathrm{~d} t}{\left(t^{2}+s^{2}+2 \bar{n}_{z} n_{\tau} t s\right)}\right], \quad 0 \leq s \leq 1,
\end{gathered}
$$

where now $n_{z}=-i e^{-i \beta / 2}$ and $n_{\tau}=i e^{i \beta / 2}$.

Finally, integrals of the type in (20) and (21), for all involved basis functions $\tau_{*}^{\lambda_{n}-1}$, $\tau_{*}^{\bar{\lambda}_{n}-1}, \tau_{*}^{\mu_{n}-1}$, and $\tau_{*}^{\bar{\mu}_{n}-1}$, and target points are precomputed adaptively to a high accuracy and stored as complex matrices. The composition of these matrices with the mapping $A$ and the pointwise values of $\Phi(\tau)$ gives $M_{3} \Phi(z)$.

\subsection{Interaction between corner panels and other panels}

The problems connected with self-interaction of $M_{3} \Phi(z)$ on a corner panel were carefully taken care of in Subsection 7.3. New problems occur when the source points of $\Phi(\tau)$ are on a corner panel and the target points are on some other panel.

First we consider target points situated on regular panels not more than a distance of $2 l_{\mathrm{cp}}$ away from the tips of a corner panel. We call these panels neighbouring panels. The action of $M_{3} \Phi(z)$ for points on neighbouring panels is calculated in the same fashion as the self-interaction in Subsection 7.3. The only difference is that $s$ is now a number $-3 \leq s \leq-1$ or $1 \leq s \leq 3$.

When target points are situated further away from the corner panel we run into problems. The computational task is to convolute the smooth kernel of $M_{3}$ with the singular basis functions of $\Phi(\tau)$. Adaptive precomputation, as for self-interaction, is of course again an option, but this procedure gets increasingly costly and complicated when we extend it too far beyond the neighbouring panels. If we were to construct a naive product integration rule for the convolution it would be, roughly speaking, only third-order accurate. This is so since the quadrature would be based on sixteen real coefficients $\Re \mathrm{e}\left\{f_{n}\right\}, \Im \mathrm{m}\left\{f_{n}\right\}, \Re \mathrm{e}\left\{g_{n}\right\}$, and $\Im \mathrm{m}\left\{g_{n}\right\}$, but only on four complex pointwise values of the kernel $M_{3}$ on each corner panel leg, see Figure 3. While the resolution of the unknown potential $\Phi(\tau)$ is sufficient, the resolution of the analytically known kernel $M_{3}$ is too poor. Fortunately, the fix is easy. We simply use temporary interpolation: The mapping $A$ gives accurate and rapidly decaying coefficients $\Re \mathrm{e}\left\{f_{n}\right\}, \Im \mathrm{m}\left\{f_{n}\right\}, \Re \mathrm{e}\left\{g_{n}\right\}$, and $\Im \mathrm{m}\left\{g_{n}\right\}$. Each basis function is interpolated at 16 temporary points on each leg of the corner panel. The kernel $M_{3}$ is evaluated at the 
temporary source points and integration is performed on the temporary mesh. For this, weights $w_{m}$ are precomputed using relations of the type

$$
\int_{0}^{1} t^{\lambda} f(t, s) \mathrm{d} t \approx \sum_{m=1}^{16} f\left(t_{m}, s\right) \int_{0}^{1} t^{\lambda} \prod_{\substack{k=1 \\ k \neq m}}^{16} \frac{\left(t-t_{k}\right)}{\left(t_{m}-t_{k}\right)} \mathrm{d} t=\sum_{m=1}^{16} f\left(t_{m}, s\right) w_{m}
$$

where $f(t, s)$ is a smooth function of its arguments, $t_{m}$ are parameter values of the temporary points, and the integration is performed by a combination of analytic and algebraic methods.

\section{Numerical results}

In this section we implement a Nyström scheme for (7), as outlined in Sections 6 and 7. We use the GMRES iterative solver [30] for the resulting system of linear equations. Compensated summation $[42,43]$ is used for the computation of matrix-vector multiplications and inner products in the GMRES iterative solver. The GMRES iterations are terminated when the norm of the residual (divided with the norm of the right hand side) is as small as it can get, which typically means $2 \cdot 10^{-16}$. We apply the algorithm to the setup in Figure 2 with notch opening angle $\beta=3 \pi / 2$, relative notch depth $a / w=0.4$ and $a / w=0.5$, and height to width ratio ranging from $h / w=1$ to $h / w=3$. We present numerical results for the notch stress intensity factor $Q_{\mathrm{I}}$ computed according to (19) and show a plot of the stress field inside the material for the case $a / w=0.5$ and $h / w=1$.

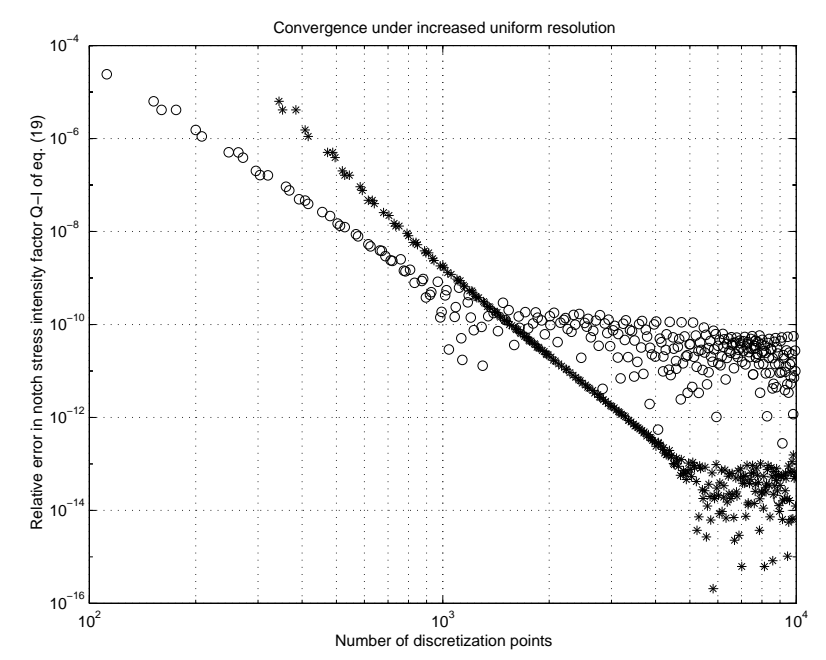

Figure 4: Convergence of the mode I notch stress intensity factor $Q_{\mathrm{I}}$ for the single edge notched specimen of Figure 2 under uniform refinement of the mesh. The notch opening angle is $\beta=3 \pi / 2$. The height to width ratio is $h / w=1$. The relative notch depth is $a / w=0.5$. The applied traction $t_{y}^{\mathrm{pr}}$ is unity. Open circles refer to a mesh where all panels have approximately the same length. Stars refer to a mesh where the panels neighbouring to corner panels have been subdivided twice. The reference value for the open circles is taken as $Q_{\mathrm{I}}=4.2958869646$. The reference value for the stars is taken as $Q_{\mathrm{I}}=4.295886967698799$. 
There are quite a number of choices that can be made in the implementation process. The most important include how to construct the mesh, what order to use for the quadrature on different panels, and how many Williams basis functions to incorporate for the different corners. Certainly, there exist optimal choices, which may depend on requested accuracy, storage capacity, the actual geometry, and speed requirements. Here we will focus on demonstrating stability and generality. To this end we use corner panels of equal length for all the seven corners of the specimen in Figure 2 and we use the same number of basis functions on all corner panels. More selective choices would, of course, reduce the degrees of freedom needed to achieve a given accuracy. In particular, fewer basis functions are needed on the panels in the four perpendicular corners $A, B, C$ and $G$ of Figure 2, than on the panel in the notch corner $E$.

\subsection{A uniform mesh}

The first computation is done for $h / w=1$ and $a / w=0.5$ and on a uniform mesh, that is, we use corner panels and regular panels of approximately equal length. The open circles of Figure 4 show that with only 112 uniformly distributed quadrature points we get a relative accuracy in $Q_{\mathrm{I}}$ of about $2 \cdot 10^{-5}$. As the mesh is uniformly refined $Q_{\mathrm{I}}$ slowly converges to about nine digits. This happens at 1000 discretization points. Very little happens as the mesh is refined further. The reason for the slow and prematurely halted convergence is that the contribution from $\Phi(\tau)$ on a regular panel neighbouring to a corner panel will not be accurately computed for target points on the corner panel. This was discussed in Subsection 7.1.

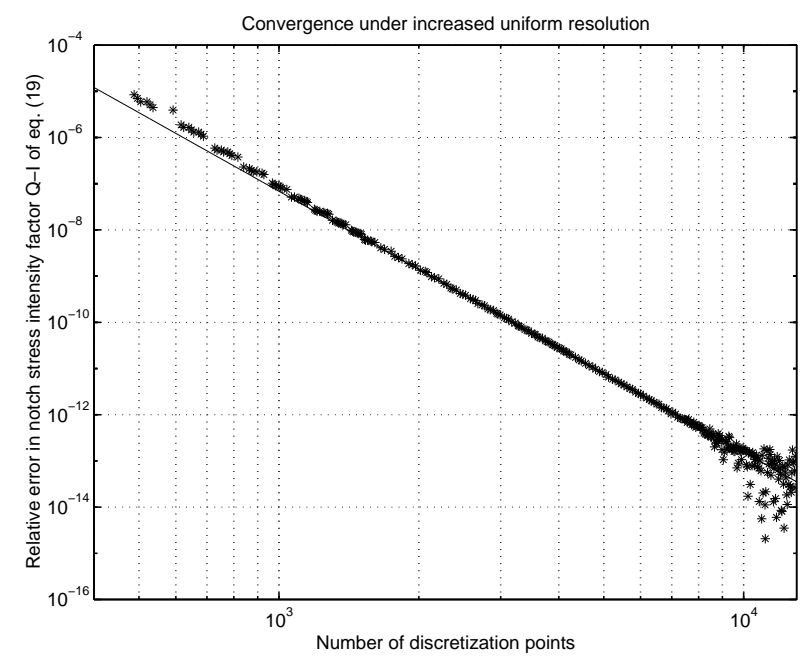

Figure 5: Convergence of the mode I notch stress intensity factor $Q_{\mathrm{I}}$ for the single edge notched specimen of Figure 2 under uniform refinement of the mesh. The notch opening angle is $\beta=3 \pi / 2$. The height to width ratio is $h / w=3$. The relative notch depth is $a / w=0.5$. The applied traction $t_{y}^{\mathrm{pr}}$ is unity. The reference value is taken as $Q_{\mathrm{I}}=4.2959268685532$. The straight line has the theoretically predicted asymptotic convergence rate $O\left(N^{-5.647}\right)$. 


\subsection{Neighbouring panels smaller than corner panels}

Next we subdivide all regular panels neighbouring to corner panels into three smaller panels. The interaction of $\Phi(\tau)$ on neighbouring panels with target points on corner panels will be more accurately computed and we get the convergence illustrated by the stars in Figure 4 . Note that the slope of this curve shows an asymptotic convergence rate of approximately $O\left(N^{-6}\right)$, where $N$ is the total number of discretization points. This, we argue, is not a coincidence. A study of the decay of the coefficients in expansions of $\Phi(\tau)$ on various parts of $\Gamma$ indicates that, under uniform refinement, the least resolved part of $\Phi(\tau)$ is on the corner panel containing the notch. This implies that the number of basis functions for the Williams series in the notch is the key factor determining the convergence rate of $Q_{\mathrm{I}}$. In the representation of (18) for $\Phi(\tau)$ in the notch we used $m_{1}=m_{2}=1$. The first omitted symmetric basis function is the one associated with the coefficient $\Im \mathrm{m}\left\{f_{5}\right\}$ and which has a Williams exponent $\lambda_{5}=5.647111773660799+0.5136838120241800 i$. The magnitude of the real part of this exponent is roughly consistent with sixth order convergence. The asymptotic convergence rate is easier to determine from a convergence plot of $Q_{\mathrm{I}}$ for a height to width ratio of $h / w=3$, see Figure 5. The agreement with the theoretical prediction $O\left(N^{-5.647}\right)$ is good.

The computing cost, in our implementation, is proportional to $N^{2}$. This is so since we form the matrix corresponding to the operator $M_{3}$ of (8) explicitly prior to solving the discretized system of equations with the GMRES iterative solver. A matrix-free implementation of the matrix-vector multiplications in the GMRES solver would reduce the complexity to $O(N)$. See Greenbaum et al. [37], Greengard et al. [38], and Greengard and Helsing [45] for examples where the so-called fast multipole method has been used for this purpose in the context of linear elasticity in 2D. As for particular timings with our present code we report the following, measured on a SUN Ultra 10 workstation for a discretization with 1056 points: Precomputation of the seven mappings $A$ of Subsection 7.2 and the quadrature weights of Subsection $7.4-5$ seconds. Computation of parts of the matrix $M_{3}$ which correspond to self-interaction on corner panels and interaction of $\Phi(\tau)$ at source points on corner panels with target points on neighbouring panels as described in Subsections 7.3 and $7.4-24$ seconds. Computation of the remaining elements of the matrix $M_{3}$ according to Subsection $7.1-8$ seconds. Performing 43 iterations with the GMRES iterative solver -73 seconds.

Remark 8.1 If only a crude estimate of $Q_{\mathrm{I}}$ for a single edge notched specimen is of interest, it is possible that the method of Gross and Mendelson [9] is more efficient than our algorithm. The implementation corresponding to open circles in Figure 4 gives a relative error in $Q_{\mathrm{I}}$ of the order of $10^{-5}$ in less than ten seconds using a SUN Ultra 10 workstation (including precomputations). No estimates of computational costs are made in Gross and Mendelson [9], but stability problems are mentioned.

\subsection{Some other geometries}

The convergence study for $Q_{\mathrm{I}}$ in Figures 4 and 5 refers to a single edge notched specimen with notch opening angle $\beta=3 \pi / 2$, relative notch depth $a / w=0.5$, and height to width 


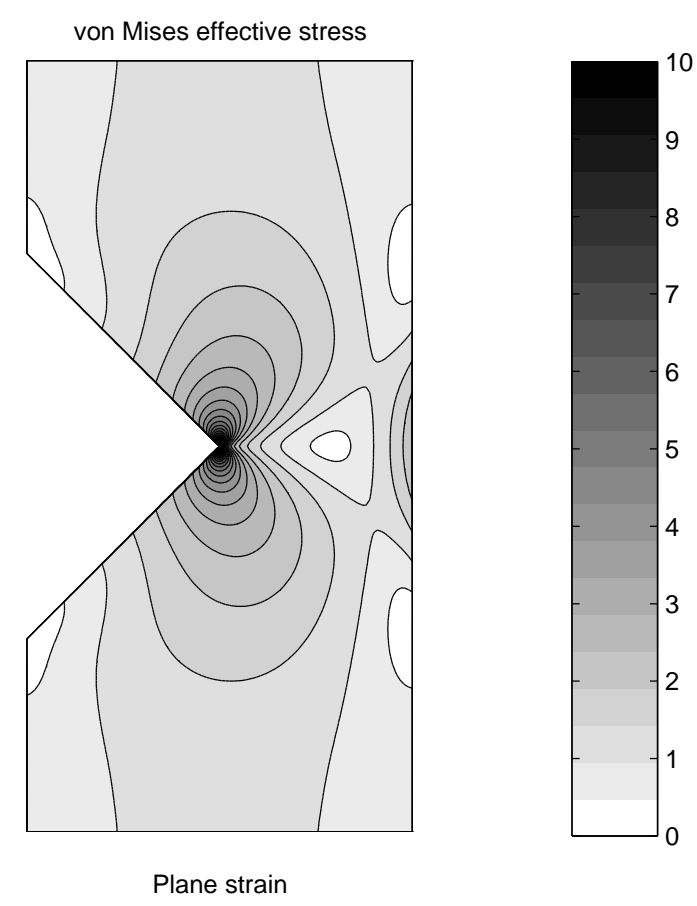

Figure 6: Distribution of the von Mises effective stress for the single edge notched specimen of Figure 2. Plane strain is assumed with Poisson's ratio $\nu=0.3$. The notch opening angle is $\beta=3 \pi / 2$. The height to width ratio is $h / w=1$. The relative notch depth is $a / w=0.5$. The applied traction $t_{y}^{\mathrm{pr}}$ is unity.

ratio $h / w=1$ and $h / w=3$. Converged values of the notch stress intensity factor $Q_{\mathrm{I}}$ for some other relative notch depths and height to width ratios are presented in Table 1 . It is interesting to note that, for the notch opening angle $\beta=3 \pi / 2$ and the relative notch depth $a / w=0.5$, the variation of $Q_{\mathrm{I}}$ with the height to width ratio $h / w$ exhibits small oscillations. While the oscillations in $Q_{\mathrm{I}}$ decay rapidly in the interval $1 \leq h / w \leq 3$ we observed a local minimum at $h / w \approx 1.1$, a local maximum at $h / w \approx 1.5$, another minimum at $h / w \approx 2.5$ and another maximum at $h / w \approx 2.9$.

\subsection{Stress fields}

Notch stress intensity factors $Q_{\mathrm{I}}$ are not the only quantities of interest which can be extracted once the solution $\Phi(\tau)$ to $(7)$ is known. The entire stress field in the body can be computed too. Pointwise values of the stress field are used in point-stress and mean-stress failure criteria and they enter into the computation of the von Mises effective stress $\sigma_{\mathrm{e}}$. The von Mises effective stress is frequently used to predict the occurrence of yielding in a material where the stress state is multiaxial. Yielding is assumed to take place when the pointwise value of $\sigma_{\mathrm{e}}$ reaches the value of a material's tensile yield strength.

Uniform convergence of $\Phi(\tau)$ is crucial for the accurate computation of stress fields close to the boundary $\Gamma$. There are no path-independent integrals or other indirect ways for the computation of stress fields, as was the case for the notch stress intensity factor $Q_{\mathrm{I}}$. The 


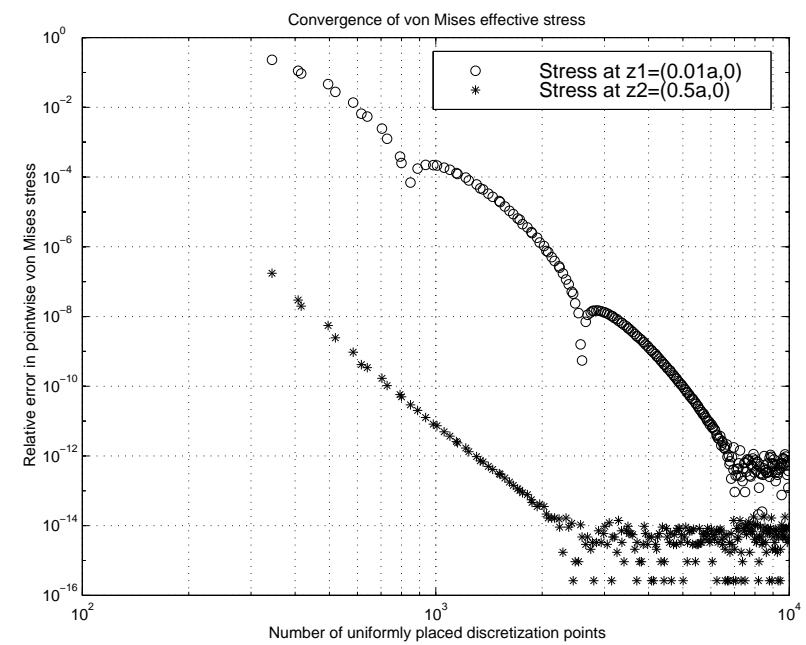

Figure 7: Pointwise convergence of the von Mises effective stress $\sigma_{\mathrm{e}}$ for the single edge notched specimen of Figure 2 at the two points $z_{1}=(0.01 a, 0)$ and $z_{2}=(0.5 a, 0)$ under uniform refinement of the mesh. Plane strain is assumed with Poisson's ratio $\nu=0.3$. The notch opening angle is $\beta=3 \pi / 2$. The height to width ratio is $h / w=1$. The relative notch depth is $a / w=0.5$. The applied traction $t_{y}^{\mathrm{pr}}$ is unity. The reference value of the stress at the two points are taken as $\sigma_{\mathrm{e} 1}=8.232225496337321$ and $\sigma_{\mathrm{e} 2}=0.4221120540554291$.

distribution of the von Mises effective stress for the case of plane strain is shown in Figure 6 and the convergence of pointwise values for the stress at the points $z_{1}=(0.01 a, 0)$ and $z_{2}=(0.5 a, 0)$ are shown in Figure 7. Plane strain is assumed in the whole specimen. This implies that $\epsilon_{z z}=0$ and $\sigma_{z z}=\nu\left(\sigma_{x x}+\sigma_{y y}\right)$, where $\nu$ is the Poisson's ratio. In Figures 6 and $7, \nu=0.3$ was used, which is a typical value for metallic materials. The assumption of plane strain is most realistic in an area close to the notch tip. An assumption of plane stress there would give infinite displacements in the thickness direction of the specimen. The von Mises effective stress, for plane strain conditions, can be expressed as

$$
\sigma_{\mathrm{e}}=\left[(1-\nu(1-\nu))\left(\sigma_{x x}+\sigma_{y y}\right)^{2}-3\left(\sigma_{x x} \sigma_{y y}-\sigma_{x y}^{2}\right)\right]^{1 / 2} .
$$

Figure 6 shows very high stress levels close to the notch tip, indicating that yielding would first occur there. High stress levels close to the edge opposite the notch can also be observed. This is due to the bending deformation of the specimen. It can be noted that there are several areas with very low stress levels, shown as white in Figure 6.

\section{Conclusions and discussion}

We have demonstrated that interior stress problems on polygonal domains can be solved in a stable way and at a reasonable computing cost. Key ingredients in our scheme are a second kind Fredholm integral equation with compact operators presented in Section 4, the use of sets of several asymptotically correct basis functions of the form derived in Section 5 for each corner, and a carefully implemented quadrature described in Section 7. This allows 
for the resolution of stress fields everywhere in the domain. Most previous authors have used some sort of "brute force" or low order accurate approaches, reviewed in Section 3. Such approaches lead to less stable and computationally more expensive schemes.

The rate of convergence and the achievable accuracy in our numerical examples are both controlled by the number of basis functions included in the expansions of the potential $\Phi(z)$ in the corners of the domain. More basis functions give a higher convergence rate but a lower achievable accuracy. We decided to use eight points for the support of $\Phi(z)$ on corner quadrature panels. This gave an asymptotic convergence rate under uniform refinement of $O\left(N^{-5.647}\right)$. The condition number for the mapping between pointwise values of $\Phi(z)$ and coefficients in the series expansion, on corner panels, was approximately $10^{3}$. An achievable relative error in the notch stress intensity factor $Q_{\mathrm{I}}$ of $10^{3} \cdot \epsilon_{\text {mach }} \approx 10^{-13}$ in IEEE Double Precision arithmetic was observed. The condition number of the underlying mathematical problem to determine $Q_{\mathrm{I}}$ is on the order of one.

One may argue that infinitely sharp corners are not physically realistic and that computations on domains with sharp corners are not of interest. One may, further, argue that it should be simpler to evaluate stress fields on domains with "rounded" corners than on domains with sharp corners. Our answer to such a criticism is as follows: While it is true that no physical corner is infinitely sharp, a sharp corner is often a good and widely used approximation of a physical corner and therefore of interest. This paper shows that it is comparatively cheap to accurately resolve stress fields on domains with sharp corners. Resolving a stress field in a domain with "rounded" corners would require more work. This is so since in a "rounded" corner the mesh must be sufficiently refined to resolve the actual rounding, while in a sharp corner the asymptotic form of the stress field is known, and the corner panel can be taken rather large.

Numerous applications, improvements, and extensions of our algorithm are possible. The application to asymmetric V-notches is straight-forward. Incorporation of the fast multipole method for linear complexity was mentioned in Section 8. Implementation of a more efficient post-processor for the evaluation of potential fields close to their sources [44, 45 ] is another option which would be economical should one want to work with failure criteria based on pointwise stress very close to, but not on, the boundary. The technique discussed in Ref. [44] can also be used in the solver to deal with boundaries that almost fold back on themselves, such as the boundary of a V-notch where $\beta \rightarrow 2 \pi$. See Strandberg [27] for an implementation in the context of the Sherman-Lauricella equation.

Another extension is to allow for cracks and inclusions in the interior of the domain. Material discontinuities may give rise to stress singularities [46]. Difficult setups with large number of cracks and smooth inclusions in infinite domains have already been successfully treated with representations of $\Psi(z)$ similar to the ones of the present paper [39, 45, 47]. Inclusions with cusps have been treated the context of Laplace's equation [44]. The presence of cracks and inclusions within a finite domain, however, may introduce extra difficulties (if one wants to stick to a single representation of $\Psi(z)$, require that the unknown quantity in the integral equation to be the limit of an analytic function, and avoid the introduction of integral operators containing arbitrarily placed points, see Remark 4.1.) Work on this problem is in progress.

Yet two extensions pertain to anti-plane conditions, such as Saint-Venant torsion and anti-plane shear, and to anisotropic materials. The problem here is reduced to solving 
Laplace's equation with Dirichlet or Neumann boundary conditions [20, 48, 49]. The solution of Laplace's equations on a polygonal domain requires a somewhat different potential representation, but is generally easier to obtain than the solution of the biharmonic equation. Integral equations based on analytic functions theory can be derived also for linear anisotropic elasticity. See pp. 34-37 of Mura [50] for an introduction and Ref. [51] for a numerical treatment of an ortotropic matrix with smooth ortotropic inclusions. The series expansion, analogous to the Williams expansion, for a notch in an ortotropic material can be found in Bogy [52].

\section{Acknowledgement}

This work was supported by NFR, TFR, and The Knut and Alice Wallenberg Foundation under TFR contracts $98-568$ and 99-380.

\section{References}

[1] Busch M, Heinzelmann M, Maschke HG. 'A cohesive zone model for the failure assessment of V-notches in micromechanical components'. International Journal of Fracture 1994; 69: R15-R21.

[2] Lin KY, and Tong P. 'Singular finite elements for the fracture analysis of V-notched plate'. International Journal for Numerical Methods in Engineering 1980; 15: 13431354.

[3] Al-Ostaz A, Jasiuk I. 'Crack initiation and propagation in materials with randomly distributed holes'. Engineering Fracture Mechanics 1997; 58: 395-420.

[4] Cleveringa HHM, Van der Giessen E, Needleman A. 'A discrete dislocation analysis of bending'. International Journal of Plasticity 1999; 15: 837-868.

[5] Seweryn A. 'Brittle fracture criterion for structures with sharp notches'. Engineering Fracture Mechanics 1994; 47: 673-681.

[6] Dunn ML, Suwito W, Cunningham S. 'Fracture initiation at sharp notches: correlation using critical stress intensities'. International Journal of Solids and Structures 1997; 35: $3873-3883$.

[7] Dunn ML, Suwito W, Cunningham S, May CW. 'Fracture initiation at sharp notches under mode I, mode II, and mild mixed mode loading'. International Journal of Fracture 1997; 84: 367-381.

[8] Strandberg M. 'Fracture at V-notches with contained plasticity'. International Journal of Fracture 2001 (in the press).

[9] Gross B, Mendelson A. 'Plane elastostatic analysis of V-notched plates'. International Journal of Fracture Mechanics 1972; 8: 267-276. 
[10] Williams ML. 'Stress singularities resulting from various boundary conditions in angular corners of plates in extension'. ASME Journal of Applied Mechanics 1952; 19: $526-528$.

[11] Helsing J. 'Corner singularities for elliptic problems: special basis functions versus brute force'. Communications in Numerical Methods in Engineering 2000; 16: 37-46.

[12] Muskhelishvili NI. Some Basic Problems of the Mathematical Theory of Elasticity, P. Noordhoff Ltd, Groningen, 1953.

[13] Carpenter WC. 'A collocation procedure for determining fracture mechanics parameters at a corner'. International Journal of Fracture 1984; 24: 255-266.

[14] Eshelby JD. 'The force on an elastic singularity'. Philosophical Transactions of the Royal Society of London 1951; A244: 87-112.

[15] Carpenter WC. 'Calculation of fracture mechanics parameters for a general corner'. International Journal of Fracture 1984; 24: 45-58.

[16] Carpenter WC. 'Mode I and mode II stress intensities for plates with cracks of finite opening'. International Journal of Fracture 1984; 26: 201-214.

[17] Carpenter WC. 'Insensitivity of the reciprocal work contour integral method to higher order eigenvalues'. International Journal of Fracture 1995; 73: 93-108.

[18] Atkinson C, Bastero JM, Martínez-Esnaola JM. 'Stress analysis in sharp angular notches using auxiliary fields'. Engineering Fracture Mechanics 1988; 31: 637-646.

[19] Sinclair GB, Okajima M, Griffin JH. 'Path independent integrals for computing stress intensity factors at sharp notches in elastic plates'. International Journal for Numerical Methods in Engineering 1984; 20: 999-1008.

[20] Givoli D, Rivkin L. 'The DtN finite element method for elastic domains with cracks and re-entrant corners'. Computers \& Structures 1993; 49: 633-642.

[21] Yosibash Z, Schiff B. 'A superelement for two-dimensional singular boundary value problems in linear elasticity'. International Journal of Fracture 1993; 62: 325-340.

[22] Barone MR, Robinson AR. 'Determination of elastic stresses at notches and corners by integral equations'. International Journal of Solids and Structures 1972; 8: 1319-1338.

[23] Lim KM, Lee KH, Tay AAO, Zhou W. 'A new variable-order singular boundary element for two-dimensional stress analysis'. International Journal for Numerical Methods in Engineering 2002; 55(3):293-316.

[24] Theocaris PS, Ioakimidis NI. 'Mode I stress intensity factors at corner points in plane elastic media'. Engineering Fracture Mechanics 1980; 13: 699-708.

[25] Portela A, Aliabadi MH, Rooke DP. 'Efficient boundary element analysis of sharp notched plates'. International Journal for Numerical Methods in Engineering 1991; 32: $445-470$. 
[26] Chen DH. 'Stress intensity factors for V-notched strip under tension or in-plane bending'. International Journal of Fracture 1995; 70: 81-97.

[27] Strandberg M. 'A numerical study of the elastic stress field arising from sharp and blunt V-notches in a SENT-specimen'. International Journal of Fracture 1999; 100: 329-342.

[28] Mikhlin SG. Integral Equations. Pergamon Press: London, 1957.

[29] Parton VZ, Perlin PI. Integral Equation Methods in Elasticity. MIR: Moscow, 1982.

[30] Saad Y, Schultz MH. 'GMRES: a generalized minimum residual algorithm for solving nonsymmetric linear systems'. SIAM Journal on Scientific and Statistical Computing 1986; 7: 856-869.

[31] Hart DJ. 'A note on the plate width and notch angle correction factors for single edge V-notched plates in tension'. Engineering Fracture Mechanics 1976; 8: 747-748.

[32] Ioakimidis NI, Theocaris PS. 'A note on stress intensity factors for single edge Vnotched plates in tension'. Engineering Fracture Mechanics 1978; 10: 685-686.

[33] Bangji Z, Fuyuan L, Qingtong W. 'Determining $K_{\mathrm{I}}$ of a V-notch from the existing results of a linear crack'. Engineering Fracture Mechanics 1988; 31: 923-929.

[34] Strandberg M. 'Upper bounds for the notch intensity factor for some geometries and their use in general interpolation formulae'. International Journal of Fracture 2001 (in the press).

[35] Murthy NS, Rao PR. 'Determination of mode 1 notch stress intensity factor by the photoelastic technique'. Engineering Fracture Mechanics 1985; 21: 557-562.

[36] Helsing J. 'On the interior stress problem for elastic bodies'. Journal of Applied Mechanics 2000; 67 658-662.

[37] Greenbaum A, Greengard L, Mayo A. 'On the numerical solution of the biharmonic equation in the plane'. Physica D 1992; 60: 216-225.

[38] Greengard L, Kropinski MC, Mayo A. 'Integral equation methods for Stokes flow and isotropic elasticity in the plane'. Journal of Computational Physics 1996; 125: 403-414.

[39] Helsing J, Peters G. 'Integral equation methods and numerical solutions of crack and inclusion problems in planar elastostatics'. SIAM Journal on Applied Mathematics 1999; 59: 965-982.

[40] Helsing J. 'On the numerical evaluation of stress intensity factors for an interface crack of a general shape'. International Journal for Numerical Methods in Engineering 1999; 44: 729-741.

[41] Helsing J, Peters G. 'An efficient numerical algorithm for cracks partly in frictionless contact'. SIAM Journal on Applied Mathematics 2000; 61: 551-566. 
[42] Kahan W. 'Further remarks on reducing truncation errors'. Communications of the Association for Computing Machinery 1965; 8: 40.

[43] Higham NJ. Accuracy and Stability of Numerical Algorithms. SIAM: Philadelphia PA, 1996; 92-97.

[44] Helsing J. 'Thin bridges in isotropic electrostatics'. Journal of Computational Physics 1996; 127: 142-151.

[45] Greengard L, Helsing J. 'On the numerical evaluation of elastostatic fields in locally isotropic two-dimensional composites'. Journal of the Mechanics and Physics of Solids 1998; 46: 1441-1462.

[46] Bogy DB. 'Two edge-bounded elastic wedges of different materials and wedge angles under surface tractions'. Journal of Applied Mechanics 1971; 38: 377-386.

[47] Helsing J. 'Fast and accurate numerical solution to an elastostatic problem involving ten thousand randomly oriented cracks'. International Journal of Fracture 1999; 100: $321-327$.

[48] Parton VZ, Perlin PI. Mathematical Methods of the Theory of Elasticity. MIR: Moscow, 1984, Vol. 1 pp. 279-302.

[49] Seweryn A, Molski K. 'Elastic stress singularities and corresponding generalized stress intensity factors for angular corners under various boundary conditions'. Engineering Fracture Mechanics 1996; 55: 529-556.

[50] Mura T. Micromechanics of Defects in Solids. Martinus Nijhoff: Dordrecht, 1987.

[51] Helsing J, Milton GW, Movchan AB. 'Duality relations, correspondences and numerical results for planar elastic composites'. it Journal of the Mechanics and Physics of Solids 1997; 45: 565-590.

[52] Bogy DB. 'The plane solution for anisotropic elastic wedges under normal and shear loading'. Journal of Applied Mechanics 1972; 39: 1103-1109. 